\title{
Importancia de la estacionalidad y del conocimiento de la demanda turística para las empresas familiares españolas
}

\section{Seasonal variation and tourist demand importance in the spanish family enterprises}

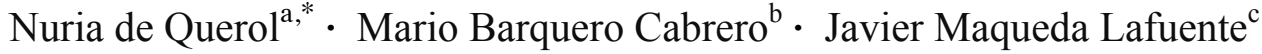 \\ ${ }^{a}$ Departamento de Fundamentos del Análisis Económico. Universidad Carlos III de Madrid, 28903 (Spain) \\ ${ }^{b}$ Departamento de Economía de la Empresas. Universidad Rey Juan Carlos, 28942 (Spain) \\ ${ }^{c}$ Economía Financiera II. Comercialización e Investigación de Mercados. Universidad del País Vasco, \\ 20018 (Spain)
}

\section{A T OS A R TÍC UL O}

\section{Historial:}

Recibido 29-12-2011

Aceptado 10-04-2012

Palabras clave:

Pernoctación

Turista

Viajero

Estancia media

Empresa familiar

Códigos JEL:

K36

\section{A R T I C LE I N F O}

Article history:

Received 29 December 2011

Accepted 10 April 2012

Keywords:

Pernoctation

Tourist

Traveller

Mean staying

Family business

JEL codes:

$\mathrm{K} 36$

\section{R E S U M EN}

Teniendo en cuesta la falta de estudios relacionados con la empresa familiar turística y considerando el interés que tiene para dicha empresa familiar el conocimiento de las preferencias del turista y saber hacer frente a la alta estacionalidad existente, hemos tratado de mostrar una visión global del estado de la demanda turística en España . El objetivo del presente trabajo es obtener conclusiones en base a los datos históricos de los diferentes turistas observando las similitudes, divergencias y el comportamiento de la estacionalidad, variables cuyo conocimiento es importante para de la empresa familiar turística. Se sugieren líneas futuras de investigación y se destaca la importancia para la empresa familiar turística de hacer frente a la alta estacionalidad existente.

\section{A B S T R A C T}

There is a lack of studies on family Business in tourism. To fill the gap, this paper focuses on identifying the factors that affect demand.

The aim of the present paper is to obtain conclusions on the basis of the historical information of the different tourists observing the similarities, differences and the behavior of the seasonal variation, all this in the environment of the tourist family enterprise. Important research gaps are identified, and future research priorities are suggested. Implications for both family business and tourism theory are discussed.

Autor de contacto.

Correoselectrónicos: nde@eco.uc3m.es,mario.barquero@urjc.es,maqueda@ehu.es 


\section{Introducción}

Las empresas turísticas familiares son una parte importante de la industria hotelera en la mayoría de los destinos turísticos (Getz y Nilsson, 2004). En España el porcentaje de empresas familiares a inicio del 2010 es superior al $70 \%$ en la oferta turística (Monfort y Camisón, 2011). El presente artículo estudia el comportamiento del turista dada la información obtenida mediante la observación y análisis del mes elegido para venir a nuestro país, centrándonos, principalmente, en el caso de los establecimientos hosteleros del sector de las denominadas empresas familiares. Es un hecho conocido la menor estancia media de los viajes, es decir, la tendencia, ya consolidada, hacia viajes más cortos y más frecuentes ${ }^{1}$. Se hace necesario, por tanto, para mantener los ingresos, que una cuantía menor en las pernoctaciones de ciertos viajeros se compense con las pernoctaciones de viajeros nuevos. Es decir, el aumento de nuevos viajeros permite compensar la disminución de las pernoctaciones de los turistas ya existentes. Conviene tener en cuenta la influencia ejercida sobre este hecho de las nuevas tendencias vigentes como son: la sobre valoración del ocio sobre el trabajo (Alegre, J y Pou, LL, 2004), la disponibilidad de recursos económicos y de tiempo libre (Porras, G. 1999), una demanda menos homogénea y más exigente que exige de la oferta una mayor diferenciación de los productos turísticos así como la tendencia creciente del porcentaje de población que realiza algún tipo de turismo (Alegre, $\mathrm{J}$ y Pou, LL, 2004).

El presente estudio se estructura de la siguiente forma: en primer lugar analizaremos el estado actual de la empresa turística familiar en España y en la literatura científica, a continuación presentaremos la metodología del estudio, los resultados y por último las conclusiones y líneas futuras de investigación.

\section{La empresa turística familiar}

La empresa familiar es una organización

${ }^{1}$ En los 20 años objeto de estudio, la estancia media disminuye de 4,5 días en 1983 a 3,7 días en 2002. social de gran complejidad, ya que en ella conviven la empresa y la familia (Díez, 2011). La investigación en la empresa familiar ha evolucionado en los últimos años, lo que puede ser apreciado en el aumento del volumen de trabajos publicados logrando abrirse camino como ámbito de estudio desde la Dirección de Empresas pero es aún una joven disciplina científica (Chrisman et al., 2008). A pesar del esfuerzo de los investigadores en la empresa familiar en desarrollar una aceptada y generalizada definición del concepto, existe aún una falta de consenso en la definición del concepto de empresa familiar (Litz, 1995; Miller et al., 2007: 832-835).

La disciplina no ha tenido un gran desarrollo hasta la última década y la literatura no es tan voluminosa como en otras áreas de la Administración de Empresas (Bird et al., 2002). Algunos temas especialmente tratados son, por ejemplo: sucesión (Lansberg \& Astrachan, 1994; Sharma, Chrisman, \& Chua, 2003), gobierno de la empresa familiar (Miller \& Le Breton-Miller, 2007) o dirección estratégica (Chrisman, Chua \& Sharma, 2005), no obstante el análisis de las particularidades de cada sector de actividad aún no ha sido ampliamente tratado. Las peculiaridades de las empresas familiares del sector turístico no han sido frecuentemente estudiadas y en general la proporción que representan las empresas familiares en el sector turístico es en términos generales amplia.

Las empresas turísticas familiares son una parte importante, si no predominante, de la industria hotelera en la mayoría de los destinos turísticos (Getz y Nilsson, 2004). Desafortunadamente, en la literatura científica se suele analizar la empresa familiar turística de forma tangencial y frecuentemente asociada al tópico del "pequeño negocio familiar", del espíritu emprendedor y temas similares (Getz y Carlsen, 2005).

Tapies (2009) analiza empresas con más de 50 millones de euros de beneficio en el sector hostelero resultando que el $64 \%$ de las empresas son familiares y suponen el $50 \%$ de la facturación así como el $50 \%$ de los puestos de trabajo generados por el total del sector. En el 
citado estudio se señala que las principales empresas del sector son familiares, por ejemplo Sol Meliá de la familia Escarrer que tiene una facturación anual de 1165 millones de euros y Barceló corporación empresarial de la familia Barceló con una facturación anual de 1004 millones de euros. Presas, Muñoz y Guía (2011) consideran el trato familiar como una de las características más importantes de las empresas familiares del sector turístico, proponiendo utilizar esta característica para diferenciarlas y mejorar su competitividad. Wanhill (1997) expone que la familia supone una aportación a la experiencia que supone el viaje para el turista y deja patente la importancia del trato familiar. De esta forma cuando la familia pasa a formar parte de su experiencia supone una atracción turística con lo que el Family branding puede convertirse en una fuente de ventaja competitiva (Wanhill,1997).

Tal y como se muestra en la tabla 1 el porcentaje de empresas familiares a inicio del 2010 es superior al 70\% en la oferta turística (Monfort y Camisón, 2011).

Tabla 1

Número de empresas familiares turísticas en España. Número

de

empresas Empleo

\begin{tabular}{lll}
\hline $\begin{array}{l}\text { Total sector } \\
\text { turístico }\end{array}$ & 440.795 & 2.143 .119 \\
$\begin{array}{l}\text { Número de } \\
\text { empresa } \\
\text { familiares }\end{array}$ & 323.567 & 1.055 .777 \\
\hline $\begin{array}{l}\text { \% empresa } \\
\text { familiar }\end{array}$ & $73,41 \%$ & $49,26 \%$
\end{tabular}

Elaboración a partir de (Monfort y Camisón, 2011).

Datos DIRCE-CNAE 2009 (a 1 de enero de 2010), EPA-INE (2009) y Cuentas Satélite del Turismo 2008

En la tabla 2 se puede apreciar la distribución de empresas familiares y no familiares en función de los distintos tipos de actividad.

Como se puede apreciar en la tabla 2 la proporción de empresas familiares es especialmente alta en transporte, restauración y alojamiento y, en general, podemos apreciar que son mayoritarias las empresas familiares en todas las actividades turísticas.

\section{Tabla 2}

Empresas familiares y no familiares según tipo de actividad.

\begin{tabular}{|c|c|c|c|}
\hline & $\begin{array}{l}\text { Empresas } \\
\text { familiares }\end{array}$ & $\begin{array}{l}\text { Empresas } \\
\text { no } \\
\text { familiares }\end{array}$ & Total \\
\hline Alojamiento & 17.156 & 5.768 & 22.924 \\
\hline Restauración & 210.036 & 56.654 & 266.690 \\
\hline Intermediación & 5.489 & 2.933 & 8.422 \\
\hline Transporte & 70.122 & 14.871 & 84.993 \\
\hline $\begin{array}{l}\text { Oferta } \\
\text { complementaria }\end{array}$ & 35.980 & 21.786 & 57.766 \\
\hline $\begin{array}{ll}\text { Total } & \text { sector } \\
\text { turístico } & \end{array}$ & 323.567 & 117.228 & 440.795 \\
\hline
\end{tabular}

Elaboración a partir de (Monfort y Camisón, 2011).

Datos DIRCE-CNAE 2009 (a 1 de enero de 2010), EPA-INE (2009) y Cuentas Satélite del Turismo 2008

Las empresas familiares son mayoritarias en todos los grupos de actividad aunque su peso relativo disminuye con el aumento del tamaño empresarial (Monfort y Camisón, 2011). Según el citado estudio el número de empresas familiares cae por debajo de la media radicalmente a partir de 50 empleados, no obstante existen aproximadamente 300 grandes empresas familiares en el sector.

Tabla 3

Empresas familiares y no familiares según tamaño.

\begin{tabular}{|c|c|c|c|}
\hline & $\begin{array}{l}\text { Empresas } \\
\text { familiares }\end{array}$ & $\begin{array}{l}\text { Empresas } \\
\text { no } \\
\text { familiares }\end{array}$ & Total \\
\hline $\begin{array}{l}\text { Empresas } \sin \\
\text { asalariados }\end{array}$ & 153.544 & 50.342 & 203.886 \\
\hline Microempresas & 168.629 & 49.184 & 217.813 \\
\hline $\begin{array}{l}\text { Pequeñas } \\
\text { empresas }\end{array}$ & 11.681 & 4.546 & 16.227 \\
\hline $\begin{array}{l}\text { Medianas } \\
\text { empresas }\end{array}$ & 1.334 & 1.000 & 2.334 \\
\hline $\begin{array}{l}\text { Grandes } \\
\text { empresas }\end{array}$ & 300 & 235 & 535 \\
\hline $\begin{array}{l}\text { Total sector } \\
\text { turístico }\end{array}$ & 335.488 & 105.307 & 440.795 \\
\hline
\end{tabular}

Como podemos apreciar la proporción que representan las empresas familiares en el sector 
turístico es en términos generales amplia, no obstante la literatura científica no es muy amplia en el caso específico de la empresa familiar turística.

\section{Metodología}

Con la finalidad de analizar la demanda turística en España, se estudiaron variables tales como el número de pernoctaciones habidas en el período objeto de estudio (1983-2008) recogidas en los establecimientos hoteleros de nuestro país, los cuales suponen más del 70\% (Devesa Martínez, T.J, 1998, IET 2002) de las elecciones del turista respecto al tipo de hospedaje elegido, quedando excluidos del estudio los habidos en formas alternativas: campings, casas de amigos, casas rurales, apartamentos o casas alquiladas. Se ha utilizado información procedente de la encuesta de ocupación hotelera, realizada por el Instituto Nacional de Estadística (INE), que tiene el objetivo de estudiar el comportamiento de una serie de variables que permitan describir la características fundamentales de la demanda del sector. A partir de dicha información, hemos elaborado una base de datos para su posterior análisis. En el presente trabajo se analizarán adicionalmente los factores estacionales de la series de pernoctaciones en España desde enero de 1983 hasta diciembre de 2008. La nacionalidad de los turistas analizados, responsables del número de pernoctaciones y cuyos comportamientos se analizan en el presente trabajo, es la siguiente: españoles, franceses, portugueses, alemanes, belgas, holandeses, británicos, luxemburgueses, italianos y estadounidenses.

\section{Resultados del estudio}

\subsection{Comportamiento observado según la nacionalidad del turista}

Podemos diferenciar los siguientes grupos de turistas según el comportamiento observado en relación a la variable objeto de estudio: el mes elegido para venir a hospedarse en nuestro país con las siguientes observaciones: a) Los turistas con una clara preferencia por los meses de verano -Junio, Julio, Agosto y Septiembre- pero con el claro liderazgo del mes de agosto, donde superan al $15 \%$ de sus pernoctaciones: los turistas españoles, italianos, portugueses, británicos, holandeses y luxemburgueses ${ }^{2}$ de los cuales solo mantienen estas preferencias sin variaciones al final del período los tres primeros.

b) Los turistas que eligen agosto con mayor intensidad, es decir los turistas más estacionales que son los italianos. portugueses y franceses, si bien los primeros con mayor énfasis al superar el $23 \%$ del número de sus pernoctaciones en este mes siendo del $19,61 \%$ en el caso de los portugueses y del $18,94 \%$ en el caso de los franceses. Estas nacionalidades muestran así no sólo su estacionalidad sino también la estabilidad de sus elecciones en los 26 años objeto de estudio superando a los españoles en esta intensidad por elegir el mes de agosto.

c) Los turistas con preferencia clara por el mes de julio en lugar de agosto y que mantienen esa elección: alemanes y belgas, superando el $16 \%$ de su elección en el último caso.

d) Los turistas que preferían agosto al inicio del período y cambian sus gustos al final del mismo eligiendo ahora en primer lugar el mes de Julio que son los británicos y holandeses.

e) Debemos destacar el cambio experimentado por los estadounidenses que al inicio del período elegían en primer lugar el mes de Julio y han cambiado en sus preferencias eligiendo al final del periodo lo sustituyen por el mes de junio.

f) Los turistas con una clara preferencia por los meses diferentes a Julio y Agosto, es decir, los menos estacionales, que es el caso de los turistas de larga distancia, en nuestro caso, los estadounidenses que prefieren en primer lugar Junio, seguido de Julio y mayo, demostrando su evolución en cuanto a la segunda preferencia en el tiempo cambiando el otoño (septiembre) por la primavera, al contrario que los holandeses

\footnotetext{
${ }^{2}$ Nótese la diferencia del comportamiento de los luxemburgueses -que se da también respecto de otras variables- con el resto de los turistas procedentes del Benelux, eligiendo los belgas y holandeses en primer lugar el mes de Julio.
} 
que sufren la evolución contraria en cuanto a segunda preferencia.

g) Los turistas que más prefieren de todos los estudiados el mes de septiembre, eligiéndolo en segundo lugar tras la elección de agosto, que son los luxemburgueses.

h) El el caso de los británicos, alemanes, estadounidenses así como los turistas provenientes del Benelux la estacionalidad es menor respecto de los españoles, siendo en cambio ésta mayor en el caso de franceses, italianos y portugueses.

Por otro lado, todos los turistas analizados han disminuido su estancia media (siendo la tasa media de decrecimiento del total general mayor al 31\%) menos los portugueses que han aumentado el número medio de días pasados en nuestro país, como se observa en la tabla mostrada a continuación:

\section{Tabla 4}

Cambios en la estancia media.

\begin{tabular}{lll}
\hline Procedencia & 2008 & 1983 \\
\hline ale & 6,31 & 9,64 \\
uk & 5,45 & 9,27 \\
bel & 5,12 & 8,58 \\
lux & 4,90 & 10,64 \\
hol & 4,58 & 9,75 \\
ita & 3,32 & 3,57 \\
fr & 2,74 & 4,24 \\
por & 2,69 & 2,02 \\
esp & 2,40 & 2,61 \\
eeuu & 2,10 & 3,16 \\
jap & 1,59 & 2,16 \\
Total & 3,24 & 4,73 \\
\hline \multicolumn{3}{l}{ Fuente: INE, elaboración propia. }
\end{tabular}

\subsection{Evolución de su comportamiento en el tiempo}

Los estadounidenses prefieren la primavera y han sustituido en el primer lugar de sus preferencias el mes de Julio en 1983 (véase tabla 4) por junio desde el año 2000. Todas las demás nacionalidades se centran en los dos meses de mayor consumo turístico: Julio y Agosto como primera o segunda opción sobre las demás opciones. Veamos las diferencias existentes entre estos dos meses, Julio y Agosto, que dan lugar a la estacionalidad temporal del sector: Julio es elegido en el 2008, preferentemente sobre el mes de Agosto sólo en el caso de los belgas y holandeses, británicos y alemanes, siendo la segunda opción para los demás turistas analizados: portugueses, italianos, franceses y españoles y estadounidenses. Belgas y alemanes muestran su estabilidad en su preferencia por Julio mientras que portugueses, italianos, franceses, españoles y luxemburgueses muestran su estabilidad en los 26 años considerados por el mes de agosto. Agosto, condicionado sin duda por las vacaciones laborales y escolares, sigue siendo el mes más elegido por la mayoría de las nacionalidades -portugueses, italianos, franceses, españoles y luxemburgueses- excepto para:

-los alemanes, británicos, belgas y holandeses que prefieren Julio.

-los británicos que lo tenían como primera opción en el pasado y han cambiado a Julio, tras Agosto y Septiembre, al igual que los holandeses aunque estos últimos prefieren mayo como tercera opción. Es destacable el hecho de que los británicos preferían Agosto a Julio en el pasado y ahora se asemejan a los alemanes eligiendo ambos Julio, Agosto y septiembre como los tres meses elegidos con preferencia sobre los demás.

\section{Tabla 5}

Evolución de las pernoctaciones de los turistas en el segundo semestre del año: mes elegido en primer lugar en 1983 y 2008.

\begin{tabular}{ll}
\hline Julio & Agosto \\
\hline BEL/1983 & POR/1983 \\
BEL/2008 & POR/2008
\end{tabular}

ITA/1983

ITA/2008

$\mathrm{UK} / 2008 \quad \mathrm{UK} / 1983$

FR/1983

HOL/2008 FR/2008

ALE/1983 ESP/1983

ALE/2008 ESP/2008

LUX/1983

EEUU/1983 LUX/2008

$\mathrm{HOL} / 1983$ 
Respecto a Septiembre, este mes era elegido como segunda opción en el pasado por italianos, portugueses y estadounidenses mientras que ahora ha pasado a ser la tercera opción para todas las nacionalidades observadas salvo los franceses y estadounidenses que la tienen en cuarta posición, mientras que los holandeses lo tienen en quinto lugar y los luxemburgueses, diferentes como siempre a sus compañeros del Benelux, lo han cambiado a su segunda opción cuando en el pasado era la tercera.

\subsection{La estacionalidad: análisis de las subseries mensuales}

Un factor decisivo en lo referente a la decisión de hacer turismo es el número de días libres reglamentado en cada país. Para comparar esta situación en los diez países de procedencia posibles se presenta la tabla 6 , de la que se deduce que dicho factor no justifica la emblemática y conocida escasa nacionalidad de los japoneses, aunque sí puede explicar la escasez de turistas estadounidenses que nos visitan. Tampoco justifica la gran concentración -sin variaciones en todos los años observadosde los italianos en el mes de Agosto, ya que dichos turistas disponen de más días de vacaciones que los alemanes y británicos.

Tabla 6

Número de días libres reglamentados en cada país.

\begin{tabular}{|c|c|}
\hline País & $\begin{array}{l}\text { Derec } \\
\text { anual } \\
\text { festiv }\end{array}$ \\
\hline España & $37-39$ \\
\hline Alemania $(\mathrm{O})$ & $29-32$ \\
\hline R. Unido & 28 \\
\hline Francia & 36 \\
\hline Italia & 32 \\
\hline Portugal & $34-36$ \\
\hline Canadá & --- \\
\hline Bélgica & 33 \\
\hline Holanda & 28 \\
\hline Luxemburgo & 35 \\
\hline Media UE & 32.8 \\
\hline Japón & 25 \\
\hline EEUU & 10 \\
\hline
\end{tabular}

Fuente: Observatorio Europeo de Relaciones Industriales (EIRO) (Organismo perteneciente a la Fundación Europea para la Mejora de las Condiciones de Vida y del Trabajo (Dublín) Información facilitada por el profesor asociado de la Universidad Carlos III de Madrid, D. Ignacio Pérez-Infante.

La tabla anterior confirma el mayor número de días retribuidos respecto a la media de la UE de Bélgica, Portugal, Luxemburgo, Francia y España, no siendo ninguno de estos países los del origen de los que más nos visitan del exterior: los turistas alemanes y británicos. No parece por tanto que la legislación que regula en cada país el número de días de vacaciones retribuidos explique convincentemente las diferencias existentes en la concentración de los viajes realizados $y$, por tanto, la estacionalidad. A continuación se ofrece la descomposición estacional, mediante las medias mensuales representadas en los gráficos de las subseries mensuales. En los gráficos la linea horizontal representa la media de las pernoctaciones de cada mes y en cada uno de los meses el valor de dicho mes en cada año, lo que permite observar cómo evoluciona a lo largo del período objeto de estudio, el comportamiento de las pernoctaciones de los turistas de las diez nacionalidades observadas desde 1983 al 2008.

\section{Gráfico 1}

Subseries mensuales de los turistas españoles, alemanes y británicos.
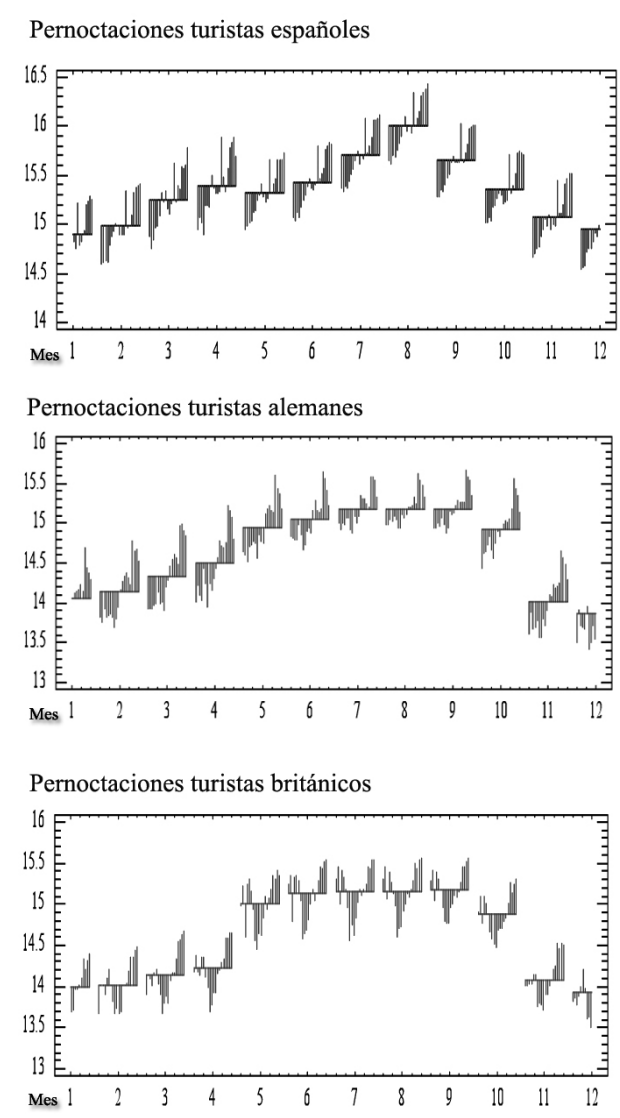
En el caso de los españoles (gráfico 1) destaca el elevado valor del mes de agosto mostrando nuestra mayor estacionalidad respecto de alemanes y británicos. Los turistas alemanes, hacen evidente en el mismo gráfico su elevado número de pernoctaciones desde el mes de mayo al de octubre, diferenciándose de los británicos que tienen el intervalo más cerrado por su menor estacionalidad aunque ésta es en ambos casos, menor a la de los españoles.

Los turistas franceses (gráfico 2) destacan por ser los menos estacionales -junto con los alemanes- de los turistas europeos que visitan nuestro país, con valores parecidos para los meses de abril, mayo, junio y julio así como con valores parecidos en septiembre respecto a mayo y junio. Por su parte los portugueses confirman el parecido con nosotros los españoles, en la concentración en los meses de julio, agosto y septiembre, aunque en menor medida que los italianos, que se confirman como los de mayor estacionalidad de todos los turistas observados debido a sus claras preferencias por el mes de Agosto.

\section{Gráfico 2}

Subseries mensuales de los turistas franceses, italianos y portugueses.

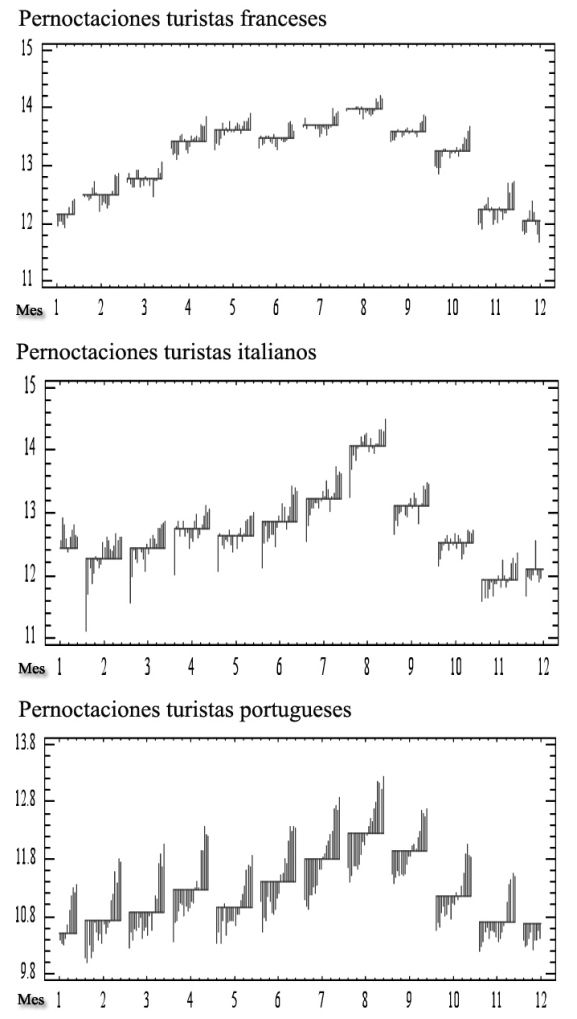

Por su parte, los estadounidenses (gráfico 3) nos visitan de forma más equitativa en el tiempo que los europeos como es propio de los turistas de larga distancia, al igual que se diferencian en su gusto por el turismo alternativo al de sol y playa, concretamente, el turismo cultural y el uso de nuestro país como "puente" hacia Europa.

\section{Gráfico 3}

Subseries mensuales de los turistas estadounidenses, belgas, holandeses y luxemburgueses.
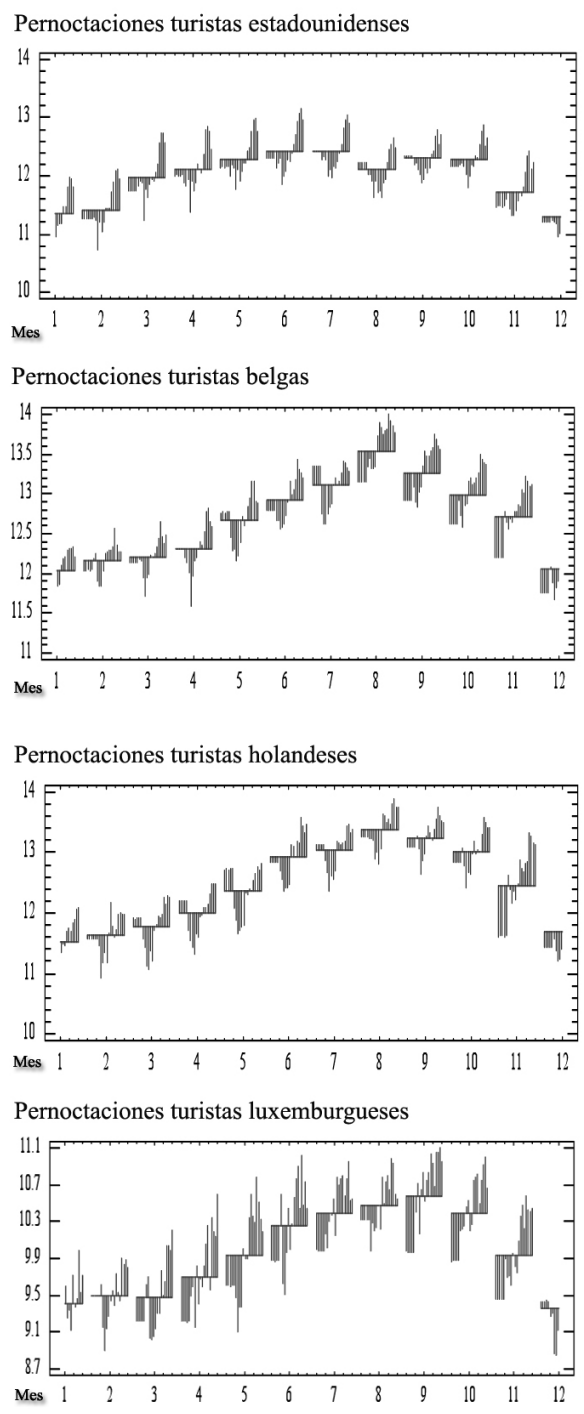

Si comparamos a los turistas franceses (gráfico 2) con los alemanes, (gráfico 1) se observa que estos últimos poseen menos estacionalidad que los primeros.

Los turistas procedentes de Luxemburgo (gráfico 3) vuelven a confirmar su comportamiento independiente respecto a sus 
compañeros del Benelux, eligiendo en menor medida Julio que los belgas y holandeses. (obsérvese la diferencia en las escalas en el eje de ordenadas entre estas tres nacionalidades) Otro dato: los holandeses gustan más del mes de mayo, lo que se muestra en el abanico más amplio.

En resumen, se confirma la menor estacionalidad de los estadounidenses, así como la menor distribución en el tiempo de portugueses, españoles e italianos, siendo los turistas que más nos visitan, británicos $\mathrm{y}$ alemanes los europeos con menor estacionalidad, y teniendo los portugueses más estacionalidad que los españoles. Asimismo es concluyente que los italianos son los turistas más estacionales de todos los observados, manteniéndose esta situación en todo el período considerado.

Los problemas derivados de la estacionalidad -es decir, de la concentración de la demanda- se puede contemplar desde dos perspectivas: la espacial y la temporal siendo ésta última la que se contempla en el presente trabajo.

El problema de la estacionalidad surge con el denominado turismo de masas, es decir, el turismo de la clase media, cuyo nacimiento estuvo sujeto tanto por el Plan de Estabilización de 1959 como por el derecho a las vacaciones pagadas surgida en 1948 (Ramón, A.B y Abellán, M.J, 1995) manifestándose por vez primera -por la aristocracia y en invierno- en ciertos destinos de la costa azul (Hartmann, R., 1986) francesa.

Por otro lado, la dependencia de los países mediterráneos de los paquetes turísticos ofrecidos a los turistas por los tour-operadores que les obligan a decidir su estancia por semanas -7 ó 14 días- sin la flexibilidad de elección de días sueltos (Alegre, J. \& Pou, L, 2002) nos perjudica enormemente ya que los turistas extranjeros que más nos visitan, británicos y alemanes ${ }^{3}$ los utilizan de forma masiva.
Igualmente, el turismo de sol y playa presenta una mayor estacionalidad en comparación con otros tipos de turismo, como el cultural, el rural ó el paisajístico y gastronómico (Viñals Blasco, M.J. \& Bernabé García, A,1999 así como Blanquer D. 2002) por ejemplo, de Galicia, y los turistas que más nos visitan siguen buscando en su mayoría el turismo del Mediterráneo, por lo que se acentúa la estacionalidad y es más difícil de erradicar.

La estacionalidad de la demanda turística está ligada directamente con su negativa consecuencia de la infrautilización de la infraestructura del sector, que obliga a cerrar las instalaciones en algún mes del año a un $17 \%{ }^{4}$ de los establecimientos de nuestro país con las repercusiones lógicas sobre el empleo, y sus consecuencias (Sancho, A, 1998), tanto a nivel nacional como sectorial e individual en un sector que ya dió empleo a más de un millón trescientas mil personas en nuestro país en el año 2004 (INE).

No hay que olvidar los efectos no solo directos sino también los indirectos (Cals, J. , 1974) del sector -debidos, por ejemplo, a las demandas generadas a los proveedores- que, a título de ejemplo sólo en Andalucía en 1996 era ya de más de 5.220 millones de euros (Aurioles, J y otros , 1998) así como el efecto "Arrastre que disminuiría nuestro PIB casi en un tercio.

En cualquier caso los mayores apoyos de la estacionalidad son los calendarios vacacionales de los trabajadores y de los centros de enseñanza (Figuerola, M, 1979) que condicionan la movilidad independientemente de los ingresos, aunque sea éste, en la forma de la renta personal disponible, junto con el precio

Comision, "Facts and figures on the europeans on holidays" (1998)

4 Exactamente un $16.92 \%$, información facilitada gentilmente por D Carlos Calvo, del IET. Madrid. De los establecimientos meramente vacacionales cierran el $80 \%$.

${ }^{5}$ El efecto arrastre es aquella parte del PIB que no se produciría si el número de turistas fuera nulo: autopistas sin usar, gasolina dejada de vender, apartamentos y hoteles sin construir...etc). Es un término específico del sector utilizado por el prof Bayón, F en una entrevista personal.

3 Todavía es mayor la dependencia de los británicos $(80 \%)$ que la de los alemanes (75\%): en European 
(medido con los precios relativos ó el tipo de cambio) los mayores determinantes (Coshall, J, 2000) del turismo.

De entre los efectos negativos provocados por la estacionalidad, cabe destacar, aparte de la baja rentabilidad de los establecimientos por la necesidad de cierre comentado anteriormente, la disminución de la calidad de los productos ofertados, la inestabilidad laboral y la menor profesionalidad de los empleados en el sector (Sancho, A, 1998).

\section{Conclusiones}

Hemos tratado de analizar el estado de la demanda turística en España considerando el interés que tiene para la empresa familiar el conocimiento de las preferencias del turista. Para la empresa familiar es importante saber que hacer frente a la alta estacionalidad existente teniendo en cuenta que ésta afectará tanto a la dimensión del negocio como a la dimensión personal de la dinámica familiar. Considerando el comportamiento de los turistas de las diferentes nacionalidades observadas a lo largo de todo el período considerado podemos extraer conclusiones del análisis realizado de los meses preferidos de los turistas, sobre la estacionalidad existente y la evolución de la estancia media.

Tras el análisis realizado para conocer el mes preferente de la demanda, se concluye:

- La dificultad de erradicar el mes de agosto como líder vacacional indiscutible sobre los demás meses del año en el caso de los turistas latinos: españoles, franceses, italianos $\mathrm{y}$ portugueses junto con los luxemburgueses.

- La fidelidad de los turistas alemanes con el mes de Julio en todo el período considerado.

-La evolución de los británicos hacia el mes de Julio como primera opción.

- Las similitudes encontradas entre británicos y alemanes en sus preferencias por elegir un intervalo más amplio para sus vacaciones así como la constancia de ambos en elegir septiembre junio y mayo como tercera, cuarta y quinta opción.
- Los turistas con mayor fidelidad mostrada por el mes de julio en todo el periodo: los alemanes $\mathrm{y}$ belgas.

- La singularidad de los turistas estadounidenses por su preferencia sobre todos los demás por el mes de junio $(12,61 \%)$ cuya evolución ${ }^{6}$ demuestra su tendencia hacia la primavera dado el tercer lugar en el ranking del mes de mayo.

Los resultados muestran la alta estacionalidad existente, y concretamente podemos verificar:

- La elevada estacionalidad de los italianos, por su concentración en el mes de agosto, seguidos de los portugueses, franceses, belgas y luxemburgueses, y nosotros mismos los españoles, en este orden, respecto a las demás nacionalidades observadas. Por tanto los portugueses eligen agosto con mayor intensidad que los españoles.

a) La menor estacionalidad de los turistas de largo recorrido: los estadounidenses, seguidos en este aspecto, respecto a los turistas procedentes de países europeos, de los belgas, holandeses, franceses, británicos y alemanes. Conviene destacar, respecto de los turistas de larga distancia, su mayor preferencia por el destino cultural, que elige varias provincias con estancias cortas de uno o dos días en cada una, mientras que el patrón de sol y playa se rige por estancias más largas.

- Las diferencias observadas entre los luxemburgueses y sus compañeros, los belgas y holandeses que siguen un comportamiento más similar tanto en cuanto a su menor estacionalidad como en cuanto al mes preferido para venir a nuestro país: julio en lugar de agosto, diferenciándose no obstante en el tercer mes elegido: septiembre los belgas y mayo los holandeses.

- La similitud entre portugueses ${ }^{7}$ y españoles, siguiendo los primeros una pauta de comportamiento parecida al turismo interior, no solo respecto de la estacionalidad sino también

\footnotetext{
${ }^{6}$ Julio ocupa el segundo lugar con un $11,76 \%$ de las pernoctaciones.

7 Los portugueses pernoctan (2008) en agosto en un $19,61 \%$ mientras que los franceses lo hacen en un $19,94 \%$ y los españoles en un $14,88 \%$.
} 
respecto otras variables como es la mayor pernoctación en provincias de interior, el uso de hoteles de categoría similar etc.

Tal y como señalan Getz y Nilsson (2004) para la empresa familiar la alta estacionalidad puede traer consigo dificultades financieras y también otras de índole personal. En la temporada alta se hace necesario un gran esfuerzo por parte de la familia lo que conduce a una alteración del tiempo de ocio y la dinámica familiar, siendo especialmente en el caso de las mujeres con niños a su cargo Getz y Nilsson (2004).

Respecto de la evolución de la estancia media de los turistas de las diez nacionalidades observadas se puede concluir:

-La mayor estancia media en nuestro país nos la ofrecen los turistas alemanes, con casi 6,31 días en el 2008 por encima todos los demás, y por tanto de los británicos ${ }^{8}$, y de nosotros mismos, los españoles.

- La relación entre la mayor estabilidad de la estancia media en los niveles superiores de renta, es decir, en las categorías superiores de hotel así como su menor estancia media en el caso de estos mismos turistas que son los de larga distancia por elegir un turismo cultural y aprovechar nuestro país para iniciar su viaje por Europa.

- Los españoles somos los que tenemos en la actualidad (2008) menor estancia media tras los turistas de larga distancia, menor, incluso a la de los portugueses, lo que no era así en el pasado, si bien somos, junto con los italianos los que poseen, respecto de este concepto la tasa de decrecimiento menor.

- A los alemanes y británicos les siguen en cuanto a la estancia media más elevada los turistas de los tres países del Benelux seguidos de los italianos, franceses, portugueses y nosotros mismos, los españoles.

- Respecto al mes con menos pernoctaciones por nacionalidad se confirma que es: Enero para los españoles y franceses, Febrero para los

\footnotetext{
${ }^{8}$ Los británicos van inmediatamente después con 6,13 días en el 2005 y 5,45 días en el 2008 de estancia media, siendo evidente la disminución general ya que en el año 2002 era de 6,7 días y en 1983 era de 9,64 días.
}

luxemburgueses, Diciembre para los belgas, holandeses, estadounidenses, británicos y alemanes y Noviembre en el caso de los italianos y portugueses.

El análisis realizado nos permite confirmar el hecho de la existencia de viajes más numerosos pero más cortos ya que la estancia media de todas las nacionalidades menos la de nuestros vecinos los portugueses, disminuye a medida que pasan los años en todo el período objeto de estudio.

\section{Limitaciones del estudio y líneas futuras de investigación.}

La principal limitación del estudio es que se ha estudiado en general la demanda turística en España, recomendándose para estudios futuros estudiar la demanda concretamente de las empresas que se pueden considerar familiares. La importancia del turismo en nuestro país junto con la vertebración de la familia y todo lo que con ella se relaciona provocan que sean considerados necesarios todos los posibles trabajos que los relacionen, yuxtaposición que escasea actualmente en las publicaciones actuales.

Por último, otra posible e interesante futura línea de investigación es aquella consistente en analizar las diferencias entre las empresas turísticas familiares y no familiares. Futuras investigaciones podrían también comparar la estacionalidad de la demanda y/o oferta entre las empresas familiares y no familiares, así como la sensibilidad (elasticidad) de la demanda turística entre establecimientos de naturaleza familiar.

Asimismo, no hay que olvidar que las áreas rurales y periféricas están especialmente dominadas por el negocio familiar (Getz y Carlsen, 2005), lo que hace necesario e imprescindible incrementar la investigación en dichas sectores.

Futuras investigaciones también podrían basarse en el estudio de la influencia de las elasticidades precio y renta en el sector turístico, que se demuestra, para el primer destino turístico de los extranjeros en nuestro país Baleares- diferente según la nacionalidad del 
mismo (Aguiló, E. \& Riera, A. \& Roselló J., , 2001) lo que explica la fidelidad de los alemanes (con elasticidad renta cercanas a cero desde los 80) mucho más indiferentes que los británicos a los cambios en su renta (para los cuales es un bien de lujo) así como en el precio 9 -con elasticidad positiva cercana a 1 en el caso de los británicos-, y cercana a 1.5 para los estadounidenses que deciden hacer un viaje de largo recorrido para venir a nuestro país (Gray, H.P. , 1970).

Aún siendo estas dos variables -precio y renta- las más influyentes en la demanda turística no hay que olvidar las especiales características de este producto que lo diferencian de los otros bienes y servicios. Así, tanto las propiedades específicas de un destino turístico -clima, paisaje, cultura- como la distancia desde el país de origen son variables ambas que aumentan la diferenciación del producto lo que le libera ligeramente de la competencia en precios.

Es del conocimiento generalizado la importancia del turismo extranjero en nuestro país pero pocos son los conocedores de la participación del turismo nacional, que supone casi el $50^{10} \%$ de las pernoctaciones y, por tanto, de los ingresos.

Las empresas turísticas familiares son una parte importante de la industria hotelera en la mayoría de los destinos turísticos (Getz y Nilsson, 2004). En España el porcentaje de empresas familiares al inicio del 2010 es superior al 70\% en la oferta turística (Monfort y Camisón, 2011).

Hemos tratado de mostrar una síntesis del estado de la demanda turística en España respecto del mes elegido para venir a nuestro país, lo que redunda en la estacionalidad existente, dada la importancia que tiene para la empresa familiar el conocimiento de las preferencias del turista y saber hacer frente a la

${ }^{9}$ La variable utilizada en lugar del precio fue el tipo de cambio, en Aguiló 2001

${ }^{10}$ Las pernoctaciones de los españoles en el total en 1983 suponen el $36,31 \%$, en el 2005 el $48,05 \%$ y en el 2008 el $48 \%$. alta estacionalidad existente, con la esperanza de beneficiar el desarrollo y consolidación del estudio de la empresa familiar turística.

\section{Bibliografía}

Aguiló E., Riera, A., y Roselló J (2001). Un modelo dinámico para la demanda turística en las Islas Baleares. Una evaluación del efecto precio el Impuesto Turístico. Palma: Universitat de les Illes Balears.

Alegre, J y Pou, LL.(2002). La reducción del tiempo de estancia en los destinos vacacionales: implicaciones sobre el gasto turístico y la estacionalidad en las islas Baleares. Madrid: Ministerio de Ciencia y Tecnología.

Alegre, J y Pou, LL. (2004). La participación de las familias españolas en el consumo turístico, Papeles de Economía Española, 24. 107 - 124.

Aurioles J. (1998). Instrumentos de observación del turismo desde una perspectiva regional.: La experiencia de Andalucía. Estudios Turísticos, 138, 57-74.

Bird, B., Welsch, H., Astrachan, J.H. \& Pistrui, D. (2002). Family business research: The evolution of an academic field. Family Business Review, 15(4), 337-350.

Blanquer, D. (2002). IV Congreso de Turismo Universidad y Empresa. Valencia: Fundació Universitat Jaume I y Tirant Lo Blanch.

Cals, J. (1974). Turismo y política turística en España: una aproximación. Madrid: Ariel.

Chrisman, J.J., Chua, J.H., Kellermanns, F.W., Matherne, C.F. III \& Debicki, B.J. (2008). Management journals as venues for publication of Family Business Research. Entrepreneurship Theory and Practice, 32(5), 927-934.

Chrisman, J.J., Chua, J.H. \& Sharma, P. (2003). Current trends and future directions in family business management studies: Toward a theory of the family firm. Coleman White Paper Series, Madison, WI: Coleman Foundation and U.S. Association of Small Business and Entrepreneurship.

Coshall, J. (2000). Análisis espectral de los Flujos Turísticos Internacionales. Annals of Tourism Research,2, 213-226. 
Devesa Martinez, P.J. (1998). Cuestiones de actualidad en el sector turístico español, Revista Valenciana d'estudis Autonómics, 25.

Díez Soto, C. M. (2011). El pago de las legítimas en dinero: un instrumento para planificar la sucesión en la Empresa Familiar. Revista de Empresa Familiar, 1(1), 23-33.

European Comision, (1998). Facts and figures on the europeans on holidays. Bruselas: European Commission Directorate General XXIIIEnterprise policy. Distributive trades, Tourism and Cooperatives

Everitt, B.S. (1993). Cluster analysis, London: Arnold.

Figuerola, M. (1979). Economía turística: Elementos de una teoría económica del turismo y métodos para su análisis cuantitativo. Madrid: sn.

Gray H.P. (1970). International TravelInternational Trade. Lexington: Heaath Lexington Books.

Hartmann, R. (1986). Tourism, seansonality and social change, Leisure Studies, 5, 25-33.

INE (2010), Número de pernoctaciones por meses: datos de los 26 años considerados -19832008- y de las 10 nacionalidades. Madrid: INE.

Instituto de Estudios Turísticos (2002). TURESPAÑA, Informe de Coyuntura. Madrid: Ministerio de Economía, Secretaría de Estado de Comercio y Turismo.

Lansberg, I. \& Astrachan, J.H. (1994). Influence of family relationships on succession planning and training: The importance of mediating factors. Family Business Review, 7(1), 39-59.

Litz, R.A. (1995). The family business: Toward a definitional clarity. Academy of Management Journal Best Papers Proceedings, 100-104.

Maqueda. F.J. (2010). Marketing, Innovación y Nuevos Negocios. Madrid: ESIC.
Miller, D. \& Le Breton-Miller, I. (2007). Kicking the habit: Broadening our horizons by studying family businesses. Journal of Management Inquiry, 16(1), 27-30.

Porras, G. (1999). Situación actual y oportunidades del turismo español. Cuadernos de Información económica, 146.

Presas, P., Muñoz, D., y Guia, J. (2011). Branding familiness in tourism family firms. Journal of Brand Management, 18 (4-5), 274-284.

Ramón, A.B. y Abellán, M.J. (1995) Estacionalidad de la demanda turística en España. Papers de Turisme, 17, 45-73.

Sancho, A. (1998) Introducción al turismo, Madrid: OMT.

Sancho, A., (1998). Educación y formación en turismo: un estudio sobre la Comunidad Valenciana Revista Valencia d'Estudis Autonomics, 25, 151164.

Sharma, P., Chrisman, J.J., \& Chua, J.H. (2003). Predictors of satisfaction with the succession process in family firms. Journal of Business Venturing, 18(5), 667-687.

Tapies, J. (2009). Empresa familiar: ni tan pequeña, ni tan joven. Barcelona: Fundación Jesús Serra.

Valls, (1999). Innovar en turismo y ocio: públicos cambiantes, nuevas satisfacciones. VIII Simposio Internacional de Turismo, ESADE Barcelona.

Viñals Blasco, M.J. y Bernabe García, A. (1999). Turismo en espacios naturales y rurales.Valencia: Universidad Politécnica de Valencia.

Wanhill, S. (1997). Peripheral Area Tourism: A European Perspective. Progress in Tourism and Hospitality Research ,3,47-70. 